\title{
COMPLETENESS OF THE WAVE OPERATORS FOR SCATTERING PROBLEMS OF CLASSICAL PHYSICS ${ }^{1}$
}

\author{
BY JOHN R. SCHULENBERGER AND CALVIN H. WILCOX
}

Communicated by Peter D. Lax, December 10, 1970

ABSTRACT. Many wave propagation phenomena of classical physics are governed by equations of the Schrödinger form $i D_{\imath} u$ $=\Lambda u$ where

$$
\Lambda=-i E(x)^{-1} \sum_{j=1}^{n} A_{j} D_{j}
$$

( $E(x)$ and the $A_{j}$ are Hermitian matrices, $E(x)$ is positive definite and the $A_{j}$ are constant.) The time-evolution of such phenomena is described by a group of unitary operators $\exp (-i t \Lambda)$ on the Hilbert space $\mathcal{H C}$ with the energy norm

$$
\|u\|_{E}^{2}=\int_{R^{n}} u(x)^{*} E(x) u(x) d x .
$$

If $E(x)$ is replaced by a constant $E_{0}$ the corresponding space and operator are denoted by $H_{0}$ and $\Lambda_{0}$. In this paper it is shown that the wave operators

$$
W_{ \pm}\left(\Lambda, \Lambda_{0}, J\right)=\operatorname{s-lim}_{i \rightarrow \pm \infty} \exp (i t \Lambda) J \exp \left(-i t \Lambda_{0}\right) P_{0}^{\mathrm{ao}}
$$

exist and are complete if $\Lambda_{0}(p)=E_{0}^{-1} \sum_{j=1}^{n} A_{j} p_{j}$ satisfies

$$
\text { rank } \Lambda_{0}(p)=m-k \text { for all } p \in R^{n}-\{0\},
$$

$E(x)$ and $D_{j} E(x)$ are continuous and bounded $(j=1,2, \cdots, n)$, $E(x)$ is uniformly positive definite, $\lim _{|x| \rightarrow \infty} E(x)=E_{0}$ uniformly in $x /|x|$ and

(5) $\int_{R^{n}}\left(1+|x|^{2}\right)^{\mu}\left|E(x)-E_{0}\right|^{2} d x<\infty \quad$ for some $\mu>n / 2$.

(In (3), $J: H_{0} \rightarrow H$ is the identification map; $J u=u$, and $P_{0}^{\text {ac }}$ is the orthogonal projection onto the absolutely continuous subspace for $\Lambda_{\mathbf{0}} . W_{ \pm}$are complete if their ranges equal $H^{\text {ac }}$, the absolutely continuous subspace for $\Lambda$.)

1. Wave operators. An abstract theory of scattering with two

AMS 1970 subject classifications. Primary 35P25, 47A40; Secondary 73D99, 76Q05, 78A45.

Key words and phrases. Symmetric hyperbolic systems, scattering theory, wave operators, absolutely continuous subspace, wave propagation, electromagnetic waves, acoustic waves, elastic waves.

1 This research was supported by the United States Office of Naval Research under Grant No. N000 14-67-A-0394-0002 and by the Swiss National Fund for Scientific Research. 
Hilbert spaces was formulated by Wilcox in [11] as an operatortheoretic description of the scattering phenomena of classical physics, and was subsequently developed by T. Kato [7], A. L. Belopol'skiǐ and M. S. Birman [2] and the authors [9]. In this theory the instantaneous states of a wave in a medium are described by the vectors of a Hilbert space $\mathfrak{H C}$ and the time-evolution of the wave is described by a group of unitary operators $e^{-i t \Delta}$ on $\mathcal{H C}$ with selfadjoint generator $\Lambda$. The scattering states of the medium are described by the vectors of $\mathfrak{H}^{\mathrm{ac}}=P^{\mathrm{ac}} \mathfrak{F C}$, the subspace of absolute continuity for $\Lambda[6, \mathrm{p} .516]$ with corresponding orthogonal projection $P^{\mathrm{ac}}$.

Scattering theory is concerned with the asymptotic behavior for $t \rightarrow \pm \infty$ of the waves in a medium. This may be studied by comparing these waves with those in a second (simpler) medium with state space $\mathfrak{H}_{0}$ and generator $\Lambda_{0}$. Let $J: \mathfrak{H}_{0} \rightarrow \mathfrak{H C}$ be a bounded linear operator which maps $\mathfrak{H}_{0}$ onto $\mathfrak{H C}$ and define the wave operators for the triple $\left(\Lambda, \Lambda_{0}, J\right)$ by

$$
W_{ \pm}\left(\Lambda, \Lambda_{0}, J\right)=\operatorname{s-lim}_{t \rightarrow \pm \infty} e^{i t \Lambda} J e^{-i t \Lambda_{0}} P_{0}^{\mathrm{ac}}
$$

when these limits exist (s-lim denotes strong limit). Each scattered wave for $\Lambda_{0}$ is asymptotically equal, for $t \rightarrow \pm \infty$, to a corresponding wave for $\Lambda$ if and only if these wave operators exist [11]. Moreover, the wave operators (1.1) map $\mathfrak{H}_{0}$ into $\mathfrak{H}$ and are partial isometries $\left[6\right.$, p. 258] with initial set $\mathcal{H}_{0}^{\mathrm{ac}}$ and final set contained in $\mathfrak{H}^{\mathrm{ac}}[2],[7]$, [11]. The wave operators are said to be complete if the final set is $\mathcal{F}^{\mathrm{ac}}$. In this case it is known [2], [7], [11] that the wave operators $W_{ \pm}\left(\Lambda_{0}, \Lambda, J^{*}\right)$ also exist and are complete, and

$$
W_{ \pm}\left(\Lambda, \Lambda_{0}, J\right)^{*}=W\left(\Lambda_{0}, \Lambda, J^{*}\right) .
$$

The principal goal of the abstract theory of scattering is to find criteria for the existence and completeness of the wave operators. The following theorem was proved by the authors in [9].

THEOREM 1.1. Let $\Lambda$ and $\Lambda_{0}$ satisfy

(1.3) $J D\left(\Lambda_{0}\right)=D(\Lambda)$,

(1.4) $J^{*} P^{\mathrm{ac}} D(\Lambda) \subset P_{0}^{\mathrm{ac}} D\left(\Lambda_{0}\right)$,

(1.5) $\Pi(\delta)\left(\Lambda J-J \Lambda_{0}\right) \Pi_{0}(\delta) \in B_{1}\left(\mathfrak{H C}_{0}, \mathfrak{H C}\right)$, and

(1.6) $\left(J^{*} J-1\right) P_{0}^{\mathrm{ac}} \Pi_{0}(\delta) \in B_{0}\left(\mathcal{H C}_{0}\right)$,

for each bounded interval $\delta \subset R^{1}$. Then the wave operators (1.1) exist and are complete.

In Theorem 1.1, $D(\Lambda)$ and $D\left(\Lambda_{0}\right)$ denote the domains of $\Lambda$ and $\Lambda_{0}$, and $\Pi(\delta)$ and $\Pi_{0}(\delta)$ their spectral measures $\left[6\right.$, p. 355]. $B_{1}\left(\mathcal{H}_{0}, \mathcal{H}\right)$ 
denotes the trace class of operators from $\mathfrak{H C}_{0}$ to $\mathfrak{H C}$ and $B_{0}\left(\mathfrak{F C}_{0}\right)$ the class of compact operators on $\mathcal{F C}_{0}[6$, p. 519]. The theorem is based on the theory of local wave operators developed by M. S. Birman [2], [3].

Theorem 1.1 was used in [9] to prove the completeness of the wave operators for a class of scattering problems of classical physics. The purpose of this announcement is to show that Theorem 1.1 can be applied to a significantly larger class of such problems.

2. Wave equations of classical physics. Many wave propagation phenomena of classical physics are governed by equations of the Schrödinger form $i D_{t} u=\Lambda u$ where $\Lambda$ is a matrix partial differential operator:

$$
\Lambda=-i E(x)^{-1} \sum_{j=1}^{n} A_{j} D_{j}
$$

$x=\left(x_{1}, x_{2}, \cdots, x_{n}\right) \in R^{n}, t \in R^{1}, D_{t}=\partial / \partial t, D_{j}=\partial / \partial x_{j}, u=u(x, t)$ is an $m \times 1$ (column) matrix, $E(x)$ and $A_{1}, A_{2}, \cdots, A_{n}$ are $m \times m$ Hermitian matrices and $E(x)$ is positive definite. Examples include electromagnetic waves, acoustic waves, elastic waves and other less familiar phenomena [11].

The matrix-valued function $E(x)$ is assumed below to have the following additional properties.

$E(x)$ is uniformly positive definite for $x \in R^{n}$; that is, the smallest eigenvalue of $E(x)$ is bounded below by a positive constant.

$$
E(x), D_{1} E(x), D_{2} E(x), \cdots, D_{n} E(x) \text { are continuous and }
$$
bounded for $x \in R^{n}$.

It follows that (if $M^{*}$ denotes the conjugate transpose)

$$
(u, v)_{E}=\int_{R^{n}} u(x)^{*} E(x) v(x) d x
$$

determines an inner product and Hilbert space $\mathcal{H C}=\{u: u(x)$ is Lebesgue measurable on $R^{n}$ and $\left.(u, u)_{E}<\infty\right\}$. Moreover, the restriction of $\Lambda$ to $C_{0}^{1}\left(R^{n} ; C^{m}\right) \subset \mathcal{H C}$ is a symmetric operator on $\mathfrak{H C}$ which is essentially selfadjoint [8]. The unique selfadjoint extension of this operator is also denoted by $\Lambda$ below.

3. The completeness theorem. Assume that

$$
\lim _{|x| \rightarrow \infty} E(x)=E_{0} \quad \text { uniformly in } x /|x|
$$


Then $E_{0}$ is positive definite by (2.2). Let $\mathfrak{F}_{0}$ and $\Lambda_{0}$ be the Hilbert space and operator (2.1) determined by $E_{0}$. Then $\mathfrak{H C}^{C}$ and $\mathfrak{K C}_{0}$ are equivalent [9] and $J: \mathfrak{K}_{0} \rightarrow \mathfrak{H C}, J u=u$ for all $u \in \mathfrak{H}_{0}$, is a bounded operator which carries $\mathfrak{H C}_{0}$ onto $\mathfrak{H}$.

The existence and completeness of $W_{ \pm}\left(\Lambda, \Lambda_{0}, J\right)$ were proved in [9] under assumptions (2.2), (2.3), (3.1),

$$
\int_{R^{n}}\left(1+|x|^{2}\right)^{\mu}\left|E(x)-E_{0}\right|^{2} d x<\infty \quad \text { for some } \mu>n / 2,
$$

and

$$
\Lambda_{0} \text { is a uniformly propagative operator. }
$$

Condition (3.3) means that the distinct eigenvalues of

$$
\Lambda_{0}(p)=E_{0}^{-1} \sum_{j=1}^{n} A_{j} p_{j}
$$

have constant multiplicity and constant algebraic sign for all $p \in R^{n}$ $-\{0\}$. The purpose of this announcement is to strengthen the completeness theorem of [9] by replacing (3.3) by the weaker condition

$$
\text { rank } \Lambda_{0}(p)=m-k \text { for all } p \in R^{n}-\{0\} \text {. }
$$

Operators $\Lambda_{0}$ with this property are said to have constant deficit $k$ [8]. The new theorem may be stated as follows.

TheOREM 3.1. The wave operators $W_{ \pm}\left(\Lambda, \Lambda_{0}, J\right)$ exist and are complete if $\Lambda, \Lambda_{0}$ and $J$ are the operators defined above, $E(x)$ and $E_{0}$ satisfy (2.2), (2.3), (3.1) and (3.2) and $\Lambda_{0}$ has constant deficit.

Condition (3.5) holds if and only if the nonzero eigenvalues of (3.4) are bounded away from zero when $|p|=1$ [10]. Thus operators $\Lambda_{0}$ with constant deficit $k$ are precisely those for which the zero eigenvalue of $\Lambda_{0}(p)$ has constant multiplicity $k$ for all $p \in R^{n}-\{0\}$. Important examples of operators with constant deficit which are not uniformly propagative occur in the theory of electromagnetic and elastic wave propagation in crystals [4, p. 602], [5]. An example which does not have constant deficit is provided by Alfvén waves in magnetohydrodynamics [4, pp. 612-617].

4. Proof of the completeness theorem. Theorem 3.1 was proved in [9], under the additional hypothesis (3.3), by using a coerciveness theorem for $\Lambda$ given in [8] and a construction of the spectral family $\Pi_{0}(\lambda)$ for $\Lambda_{0}$ given in [12] to verify hypotheses (1.3)-(1.6) of Theorem 1.1. These results were based on (3.3). The proof given in [9] 
also suffices to prove Theorem 3.1 if the coerciveness theorem of [10], based on (3.5), is used together with the following results.

THEOREM 4.1 . Let $\lambda_{1}(p) \geqq \lambda_{2}(p) \geqq \cdots \geqq \lambda_{m}(p)$ be the repeated eigenvalues of $\Lambda_{0}(p)$, ordered as indicated for all $p \in R^{n}$. Then each $\lambda_{j}(p)$ is continuous on $R^{n}$. Moreover, there exist $m$ functions $r_{j}: R^{n} \rightarrow C^{m}$ which are Lebesgue measurable on $R^{n}$ and satisfy $\Lambda_{0}(p) r_{j}(p)=\lambda_{j}(p) r_{j}(p)$ and $r_{j}(p) * E_{0} r_{k}(p)=\delta_{j k}$ for $1 \leqq j, k \leqq n$ and almost all $x \in R^{n}$.

THEOREM 4.2. The spectral family for $\Lambda_{0}$ can be written $\Pi_{0}(\lambda)$ $=\Phi^{*} \Pi_{0}(\lambda) \Phi$ where $\Phi$ is the Fourier transform on $\mathfrak{F}_{0}$ with inverse $\Phi^{*}$ and (if $H(\lambda)$ is the Heaviside function)

$$
\hat{\Pi}_{0}(\lambda)=\sum_{j=1}^{m} H\left(\lambda-\lambda_{j}(\cdot)\right) r_{j}(\cdot) r_{j}(\cdot) * E_{0}
$$

Theorem 4.1 was proved by Wilcox in [13]. Theorem 4.2 was proved by Wilcox in [12] under the additional hypothesis (3.3). It was proved by G. S. S. Avila [1] without this hypothesis on the assumption that Theorem 4.1 was true. Hypothesis (3.5) is not needed for Theorems 4.1 and 4.2 .

Hypothesis (3.5) is used in [10] in the proof of the coerciveness theorem and again, in the proof of (1.5) and (1.6), to show that the nonzero eigenvalues of $\Lambda_{0}(p)$ are bounded away from zero (see the proof of Lemma 5.2 in [9]).

\section{REFERENCES}

1. G. S. S. Avila, Spectral resolution of differential operators associated with symmetric hyperbolic systems, Applicable Analysis (to appear).

2. A. L. Belopol'skir and M. S. Birman, The existence of wave operators in the theory of scattering with a pair of spaces, Izv. Akad. Nauk SSSR 32 (1968), 1162-1175 = Math. USSR Izv. 2 (1968), 1117-1130. MR 38 \#7377.

3. M. S. Birman, Local criteria for the existence of wave operators, Izv. Akad. Nauk SSSR 32 (1968), 914-942 = Math. USSR Izv. 2 (1968), 879-906. MR 40 \#1810.

4. R. Courant and D. Hilbert, Methods of mathematical physics. Vol. 2: Partial differential equations, Interscience, New York, 1962. MR 25 \#4216.

5. G. F. D. Duff, The Cauchy problem for elastic waves in an anisotropic medium, Philos. Trans. Roy. Soc. London Ser. A 252 (1960), 249-273. MR 22 \#2157.

6. T. Kato, Perturbation theory for linear operators, Springer-Verlag, New York, 1966. MR $34 \# 3324$.

7. - Scattering theory with two Hilbert spaces, J. Functional Anal. 1 (1967), 342-369. MR 36 \#3164.

8. J. R. Schulenberger and C. H. Wilcox, Coerciveness inequalities for nonelliptic systems of partial differential equations, Ann. Mat. Pura Appl. (1971) (to appear).

9. - Completeness of the wave operators for perturbations of uniformly propagative systems, J. Functional Anal. (to appear). 
10. - A coerciveness inequality for a class of nonelliptic operators of constant deficit, ONR Technical Summary Report \#8, University of Denver, Denver, Colo., 1970.

11. C. H. Wilcox, Wave operators and asymptotic solutions of wave propagation problems of classical physics, Arch. Rational Mech. Anal. 22 (1966), 37-78. MR 33 \#7675.

12. - Transient wave propagation in homogeneous anisotropic media, Arch. Rational Mech. Anal. 37 (1970), 323-343.

13. —- Measurable eigenvectors for Hermitian matrix-valued polynomials, J. Math. Anal. Appl. (to appear).

Institut de Physique Théorique, Ecole de Physique, Boulevard d'Yooy 32, Geneva 4, Switzerland

University of Denver, Denver, Colorado 80210

University of Utah, Salt Lake City, Utah 84112 